Besitzern mit ins Grab gegeben wurden, sind die Reste jener Originalschriften. Im Bereich der griechischen Welt sind sie mit Stumpf und Stiel ausgerottet worden.

\title{
3. Sprache.
}

Die Sprache des Cod. Askewianus ist das klassische Sahidisch, resp. der oberägyptische Dialekt mit zum Teil noch altertümlichen Formen. Freilich ist Leipoldt der Ansicht, daß der Cod. Askewianus wie der Cod. Brucianus nicht das in der Thebais, sondern das im Gaue von Achmim gesprochene Sahidisch enthalte, ${ }^{1}$ aber darin kann ich ihm nicht beistimmen. ${ }^{2}$

Viel umstritten ist die Frage, ob die Pistis Sophia die Übersetzung eines griechischen Originalwerkes bildet oder von Anfang an in koptischer Sprache abgefaßt ist. Woide trat zunächst für ein griechisches Original ein, da er in Valentin den Verfasser sah, aber er stellte zugleich die Behauptung auf, Valentin als geborener Ägypter wäre sowohl des Griechischen wie des Ägyptischen mächtig gewesen und habe behufs Verbreitung seiner Lehren das Werk in beiden Sprachen verfaßt. Diese Hypothese scheitert schon an der Tatsache, daß zur Zeit Valentins um 140 n. Chr. die koptische Schrift noch gar nicht eingeführt war. Neuerdings ist aber von englischen Gelehrten die These von einem koptischen Original wieder aufgenommen. So z. B. schreibt F. Granger im Journal of Theolog. Studies, 1904, p. 401: I am surprised at the confidence with which Schmidt declares the Pistis Sophia and other Gnostic works to be translations from Greek originals. Nach ihm the Egyptian Gnostic writings of the third centary exhibit the same qualities of style as the Coptic biographies and apocalypses of the fourth and following centuries. Hatte Rendel Harris bei seiner ersten Publikation der Oden Salomos p. 35 geschrieben:

The Pistis Sophia, in which the Odes are imbedded

1 Vgl. Theol. Literaturztg. 1905, Kol. 517 and ZDMG. 58 [1904], S. 922, Anm. 6.

2 Vgl. meine Bemerkungen zum Dialekt der Pistis Sophia in der Z. f. ägypt. Sprache, 42 [1905], S. $139 \mathrm{f}$. 
dates from the third centary, and the author of the $\mathrm{P}$ is tis had, as we have shown, the Odes bound up with his Canonical Psalter; at the time intimated there was no Coptic [Thebaic] Bible from which the extracts could have been made; so we may be sure the Odes were taken from a Greek Bible, and with almost equal certainty, that the Pistis Sophia itself was a Greek book.

- so hat er in seiner großen Publikation ${ }^{1}$ seine Ansicht wie folgt geändert, wenn er schreibt:

Unless .. the P. S. has substituted the Sahidic [Bible] version for some other version which lay before the author, of which he has avoided the trouble of making a fresh translation, there is a strong presumption that the P. S. is a genuine Coptic book, and not a rendering of some other work (Greek or Syriac) into Coptic.

L e g ge, Introd. p. IX, möchte dem Urteil von Granger beitreten, particularly with regard to the Fourth and Fifth Documents, aber er fügt hinzu: but the belance of expert opinion is distinctly against this view. Hätte er den Artikel von F. C. Burkitt im Journal of Theol. Studies, 1922 (Vol. XXIII) p. 271 bei Gelegenheit einer Anzeige von Meads Buch gekannt, würde er sich mit größerer Sicherheit für den original-koptischen Ursprung ausgesprochen haben. Burkitt ist nämlich der Meinung, that the question of the language in which our Pistis Sophia was composed is still open, and er versucht an einigen Pankten zu zeigen, that our book, as it stands, has an Egyptian, non Greek origin. Als Beweis führt er folgendes an:

1. Von den 37 Sünden, vor denen die Menschen p. $256 \mathrm{ff}$. (S. 186, 27 ff.) gewarnt werden, werden nur 10 ganz oder teilweise durch griechische Wörter ausgedrückt, während die übrigen 27 in koptischer Sprache wiedergegeben werden.

2. Die griechischen Namen der fünf Planeten p. 356, $12 \mathrm{f}$. (S. 264, 2 f.): Zeus, Hermes usw. scheinen ganz frei von irgendwelcher Verbindung mit dem Götzendienst. Aphrodite wird identifiziert mit der Bubastis (p. 362, $21=$ S. 268, 36) und Zeus

1 R. Harris und A. Mingana: The Odes and Psalms of Solomon. 2 Vol. 1912 und 1920. 
wird ein „guter" genannt (p. 357,4=S. 264, 21). Dies hält B. für unmöglich in einem griechischen Text vor dem Nicaenum.

3. Der Name Pistis Sophia ist eine barbarous formation, ob sie geschrieben ist „die Pistis Sophia“ oder „die Pistis, die Sophia" (p. 356, 25 = S. 264, 14), und daraufhin stellt B. die Frage: In there any real analogy for it in any of the names of the Aeons excogitated by Greek-speaking thinkers?

Burkitt scheint nicht in Erwägung zu ziehen, daB das gnostische Werk nach allgemeiner Ansicht auf dem Boden Ägyptens entstanden ist und $\mathrm{da} B$ sich darauf die mancherlei Eigentümlichkeiten zurückführen. Ist aber Ägypten das Geburtsland, so brauchen wir in dem Verfasser keinen Hellenen im eigentlichen Sinne, sondern eventuell nur einen sogenannten Levantiner mit griechischer Sprache zu sehen.

Es sind doch ebenfalls aus diesen synkretistischen Kreisen die griechischen Zauberpapyri geflossen, die so manche Berührungspunkte, insonderheit mit dem vierten Buche der Pistis Sophia aufweisen. Und um nur auf den letzten Einwand von B. betreffend die barbarische Bildung des Namens Pistis Sophia einzugehen, so gibt uns darüber das noch unedierte Apokryphon Johannis im Cod. Berolinensis die gewünschte Auskunft. Denn dort lesen wir an einer Stelle: „Seine Männlichkeit, welche Sotêr, der Erzeuger aller Dinge, seine Weiblichkeit aber, welche Sophia $\pi \alpha v \gamma \varepsilon v \varepsilon \tau \varepsilon i \varrho a$ genannt wird, die einige ,die Pistis' heißen." Da haben wir des Rätsels Lösung. Im 2. Jahrhundert führte die Sophia bei einigen Gnostikern derselben Richtung auch den Namen „Pistis“. "Die Pistis Sophia" 1 ist also eine Zusammenstellung zweier Namen, die ursprünglich ein und dieselbe Aeonenfigur bezeichneten, was in der späteren Zeit aber kaum mehr verstanden warde. Freilich der Verfasser des 4. Buches zeigt noch ein BewuBtsein von dieser Tatsache, da er die beiden Namen trennt, wenn er "die Pistis, die Sophia“ (S. 264, 17) schreibt, wiederum ein neues Zeugnis für das höhere Alter dieses Buches. ${ }^{2}$

1 Damit ist auch die falsche Übersetzung von nla Fidèle Sagesse" bei Dulaurier und Renan erledigt.

2 Freilich auch in Buch I und II kommt neben Pistis Sophia ohne jede Unterscheidung sehr häufig Sophia vor (vgl. u. a. S. 32, 28; 33, 22 ; 
Aber m. E. führen allgemeine Beobachtungen überhaupt zu keiner Lösung der Frage, ob original-koptisch oder original-griechisch; einzig und allein der vorliegende Text $\mathrm{muB}$ darauf untersucht werden, ob wirklich eine Übersetzang vorliegt oder nicht. Ich habe an der Hand der neuen Ausgabe Material nach dieser Richtung gesammelt und bin in meiner Ansicht der Übersetzung eines griechischen Originals von neuem bestärkt worden, aber es würde die Diskussion über dieses Material an dieser Stelle zn weit führen; daher hoffe ich es in Kürze den Interessenten in einer besonderen Abhandlung vorlegen zu können. Nur kurz möchte ich schon hier zur Bestätigung meiner These auf folgendes aufmerksam machen. An zwei Stellen der Pistis Sophia (p. $253,16=\mathrm{S} .184,25$ und p. $352,20=\mathrm{S} .260,32$ ) sind uns

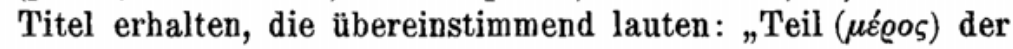

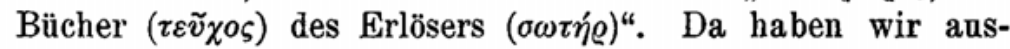
schließlich in dem Titel griechische Ausdrücke vor uns, besonders der terminus technicus $\tau \varepsilon \tilde{v} \chi o \varsigma$ "Rolle" aus dem griechischen Buchwesen ist interessant, da für diesen Ausdruck der Kopte kein Äquivalent hatte. Und hätte überhaupt ein Kopte von Haus aus einen solchen Titel aus drei griechischen Worten gebildet? Sicherlich hätte er sich ein großes Armutszeugnis ausgestellt. Wo er in seiner Vorlage das Wort $\beta i \beta \lambda$ os wie S. 179, 24. 33; 258, 14 „die beiden Bücher Jeû" fand, hat er das koptische Wort žoome eingesetzt. Noch viel evidenter für ein griechisches Original spricht eine andere Tatsache, wenn Burkitt auf die griechischen Namen der fünf Planeten hinweist. Denn ausdrücklich betont der Verfasser S. 264, $1 \mathrm{f}$., dab diese in der ganzen Menschenwelt mit den Namen Kronos, Ares, Hermes, Aphrodite und Zeus genannt werden, während ihre unvergänglichen Namen der Reihe nach Orimûth, Munichunaphôr, Tarpetanûph, Chôsi, Chônbal lauten (S. 264, 33 f.). Von dem kleinen Sabaoth, dem Guten von der Mitte, wird wiederholt bemerkt, daß er a uf Erden Zeus genannt wird (S. 268, 34; 269, 28; 271, 17);

77,$4 ; 83,18 ; 97,20 ; 107,6 ; 108,23.26 ; 109,5 ; 130,10 ; 131,10.14$; 133,19 etc.) - Pistis allein S. 121, 4. Der Name Sophia war der ursprüngliche, da in fast allen gnostischen Systemen der Fall der Sophia eine bedeutende Rolle spielte. 
desgleichen soll die Bubastis a uf Erden Aphrodite heißen (S. 268, 37; 270, 22). Ferner tragen auch die Gestirne des Zodiakalkreises lauter griechische Namen; von dem ersten Aeon der Sphaera heißt es, daß er a uf Erden xoıs genannt wird (S. 268, 2 f.). Was ist nun unter den Worten ,auf Erden“, resp. wörtlich „in der Welt $(\varkappa o ́ \sigma \mu o \varsigma)$ “ zu verstehen? M. E. doch nicht anderes, als daß der Verfasser diese Gestirne stets mit griechischen Namen benannte und sie ihm eben in dieser Form im alltäglichen Leben gebräuchlich waren. Ein geborener Kopte hätte sich niemals so ausdrücken können, d. h. die Welt mit dem Griechentum identifiziert.

Erwähnen will ich noch, daß neben M ead l. c.p. XXVI sq. sich A mélinea n besonders stark für ein griechisches Original eingesetzt hat, wenn er 1. c. p. X schreibt:

C'est ma ferme conviction que nous nous trouvons en présence d'un ouvrage traduit du grec en copte. Quiconque a quelque connaissance de la langue copte sait que cette langue ignore les longues phrases, que c'est une langue éminemment analytique et non point synthétique, que les phrases procédent toujours par petits membres très clairs, presque indépendants les uns des autres. Evidemment, tous les auteurs ne sont pas également faciles, certains d'entre eux même sont d'une grande difficulté d'intelligence; mais ce qu'il y a de certain, c'est que jamais, au grand jamais, nous ne rencontrons en copte ces périodes à incises compliquées à trois ou quatre membres différents, dont les éléments sont unis les uns aux autres d'une manière synthétique, si bien que l'intelligence de la phrase entière ne peut être obtenue qu'avec le dernier mot. Eh bien, c'est ce que le lecteur rencontrera précisément en cet ouvrage. Les phrases sont tellement enchevêtrées de propositions incidentes et compliquées, que souvent, très sonvent, le traducteur copte a perdu le fil, comme on dit, qu'il a fait des propositions incidentes les propositions principales, et que l'on trouve trois ou quatre pages plus loin la continuation de la proposition premiére ..... D'ailleurs, s'il pouvait raisonnablement rester un doute dans un esprit quelconque, l'emploi de la terminologie grecque fort touffue dans l'ouvrage serait une preuve convaincante que l'ouvrage a été primitivement écrit en grec. 